\title{
FHOD1 is upregulated in gastric cancer and promotes the proliferation and invasion of gastric cancer cells
}

\author{
CHENGFEI JIANG $^{1 *}$, BINBIN YUAN ${ }^{1 *}$, BO HANG $^{2}$, JIAN-HUA MAO $^{2}$, XIAOPING ZOU ${ }^{1 *}$ and PIN WANG ${ }^{*}$ \\ ${ }^{1}$ Department of Gastroenterology, Nanjing Drum Tower Hospital, The Affiliated Hospital of \\ Nanjing University Medical School, Nanjing, Jiangsu 210008, P.R. China; ${ }^{2}$ Biological Systems and \\ Engineering Division, Lawrence Berkeley National Laboratory, Berkeley, CA 94720, USA
}

Received October 28, 2020; Accepted June 24, 2021

DOI: $10.3892 / \mathrm{ol} .2021 .12973$

\begin{abstract}
Gastric cancer (GC) is one of the main causes of cancer-associated morbidity and mortality worldwide. The present study aimed to investigate the role of the gene encoding formin homology 2 domain containing 1 (FHOD1) protein in GC development. Data from The Cancer Genome Atlas were firstly analyzed, and immunohistochemistry was conducted on GC tissues. The results demonstrated that FHOD1 expression in GC tissues was significantly increased compared with adjacent non-tumor tissues. Furthermore, the expression level of FHOD1 was negatively associated with the overall survival of patients with GC. For the functional studies, lentivirus-mediated short hairpin RNA against FHOD1 and FHOD1-overexpression vectors were constructed to knockdown and overexpress the expression level of FHOD1 in human GC cell lines, respectively. The results indicated that FHOD1 knockdown inhibited the proliferation, colony formation and migratory and invasive abilities of GC cells. Conversely, overexpression of FHOD1 in GC cells promoted soft-agar colony formation and migratory and invasive abilities. In addition, it was demonstrated that genes of which expression levels were correlated with FHOD1 were enriched in the Gene Ontology term of 'extracellular matrix (ECM) structural constituent', suggesting that FHOD1 may serve an important role in the regulation of ECM. In conclusion, the present study demonstrated that FHOD1 may exert an oncogenic role in cultured GC cells and be inversely associated with the overall survival of patients with GC.
\end{abstract}

Correspondence to: Dr Xiaoping Zou or Dr Pin Wang, Department of Gastroenterology, Nanjing Drum Tower Hospital, The Affiliated Hospital of Nanjing University Medical School, 358 Zhongshan Road, Nanjing, Jiangsu 210008, P.R. China

E-mail: 13770771661@163.com

E-mail: pinwang729@126.com

*Contributed equally

Key words: FHOD1, gastric cancer, overall survival, overexpression, short hairpin RNA, proliferation, invasion

\section{Introduction}

Gastric cancer (GC), a malignant type of tumor originating from gastric epithelial cells, is one of the leading causes of cancer-associated mortality worldwide. Its incidence ranks fifth among all types of cancer worldwide, behind lung, breast, colorectal and prostate cancers $(1,2)$. With progress in science and biotechnology, the early diagnosis of GC has improved, and a significant increase in the survival rate of patients has been observed. However, the overall 5-year survival rate of patients with GC remains $\sim 30 \%$ due to the recurrence and metastasis of advanced GC. Furthermore, local recurrence and distant metastasis are the most common causes of death $(3,4)$.

The survival and prognosis of patients with GC have been greatly improved with the use of chemotherapy, radiotherapy, surgery, molecular targeted therapy and immunotherapy; however, the underlying molecular mechanisms for this disease remain largely unknown $(5,6)$. The carcinogenesis of GC involves both environmental and genetic factors and is a multi-step process $(7,8)$. It is therefore crucial to study the genes involved in tumor progression, invasion and metastasis, as well as to determine the underlying mechanisms of these genes in affecting both the treatment and prognosis of GC $(9,10)$. For example, efforts are currently being made to identify molecular regulators for cancer invasion.

One approach to identify the molecular regulators of GC is to search for actin regulatory proteins, which may be targets for future anticancer therapy (11). Among a variety of cellular processes, the formin family coordinates the rearrangement of the actin cytoskeleton. Actin cytoskeleton remodeling is crucial in cell migration and is mediated by actin regulatory proteins, which are active in different cellular locations (12). Formins, which are highly conserved actin nucleating proteins, are usually found in all eukaryotes. Individual formins are usually inactive, until they are activated or phosphorylated by rhodopsin (Rho) GTPases. The latter have a variety of biochemical and functional properties in the formation of actin filaments, such as remodeling the cytoskeleton of different intercellular compartments and controlling the assembly of stress fibers, the formation of adhesion points and the movement of cancer cells $(13,14)$. However, the role of formins in tumor tissues remains unknown (15). Several proteins of the formin family, including formin homology 2 domain 
containing protein 1 (FHOD1), are primarily expressed in the mesothelial tissue. It has been reported that their upregulation occurs during cancer cell epithelial-mesenchymal transition (EMT) in vivo (16). For example, the high expression of formin like 2 in colon cancer tissues is associated with metastasis (17). Despite these findings, there is a lack of studies evaluating the clinicopathological significance of FHOD1 in GC and its underlying molecular mechanisms.

The present study analyzed a dataset from The Cancer Genome Atlas (TCGA) and performed immunohistochemistry (IHC) on GC tissues to evaluate FHOD1 expression in GC tissues. Furthermore, the current study determined the association between FHOD1 expression and the overall survival rate of patients with GC. The possible role of FHOD1 in the development of GC was studied using several in vitro assays performed on two human GC cell lines that underwent FHOD1 knockdown and overexpression. The present study may provide novel evidence regarding the role of FHOD1 in GC pathogenesis and patient prognosis. FHOD1 may represent a potential new target for the treatment and management of $\mathrm{GC}$.

\section{Materials and methods}

TCGA datasets. The RNA sequencing data of FHOD1 in normal gastric and GC tissues were obtained from TCGA-STAD (https://gdc-portal.nci.nih.gov/projects/TCGA-STAD). The expression level of FHOD1 between normal gastric and GC tissues were compared. The gene expression data used to analyze the association between FHOD1 expression and the overall survival rate of patients with GC were obtained from our previous cohort study (Nanjing Drum Tower Hospital; Nanjing, China) on the multigene prognostic signature in GC (18).

Patients and tissues. To investigate FHOD1 expression in GC samples using IHC, a total of 30 patients $(76.7 \%$ men; $23.3 \%$ women; mean age, 59.63 years; age range, $33-80$ years) were enrolled between December 2012 and April 2014 at Nanjing Drum Tower Hospital. The paraffin-embedded slides, including 30 pairs of GC and adjacent gastric tissues (3-6 cm from the tumor), were obtained from the Department of Pathology of Nanjing Drum Tower Hospital. The protocol was approved by The Institutional Ethical Committee of The Nanjing Drum Tower Hospital (approval no. 2019-196-01) and signed informed consent form was obtained from each participant prior to the study.

$I H C$. Specimens were collected from GC resection following informed consent from patients. Full thickness gastric tissues (size, $1.5 \times 1.5 \times 0.3 \mathrm{~cm}$ ) were resected from the lesion and the normal section of patients' stomach. These tissues were immediately fixed in $10 \%$ neutral formalin at room temperature for $23 \mathrm{~h}$, dehydrated in ethanol solution with a concentration gradient $(50 \%$ ethanol for $10 \mathrm{~h}, 70 \%$ ethanol for $8 \mathrm{~h}$ and $100 \%$ ethanol for $1 \mathrm{~h}$, all steps performed at room temperature) and permeabilized three times in xylene (100\% xylene for $40 \mathrm{~min}$ at room temperature). Finally, the tissues were embedded in paraffin. Representative 4- $\mu \mathrm{m}$ serial sections were prepared from the tissue blocks for IHC. After deparaffinization, sections were exposed to $3 \%$ hydrogen peroxide at room temperature for $10 \mathrm{~min}$ to block endogenous peroxidase activity, and heat-mediated antigen retrieval was performed using Tris/EDTA buffer ( $\mathrm{pH} 8$ ). The FHOD1 antibody (cat. no. ab73443; polyclonal; 1:350; anti-mouse; Abcam) diluted in QuickBlock ${ }^{\mathrm{TM}}$ Primary Antibody Dilution Buffer (cat. no. P0262; Beyotime Institute of Biotechnology) was then incubated overnight with the human tissue sections in a humidified chamber at $4^{\circ} \mathrm{C}$, followed by incubation with $100 \mu 1$ of the HRP-conjugated goat anti-mouse IgG secondary antibody working solution from the Mouse two-step kit (cat. no. PV-6002; OriGene Technologies, Inc.) at $37^{\circ} \mathrm{C}$ for $30 \mathrm{~min}$. Both negative (without the primary antibody) and positive controls (human pancreatic tissue) were conducted in each run. Based on the antibody staining, a professional pathologist interpreted the IHC staining results. Images were acquired using a EVOS M7000 imaging system (Thermo Fisher Scientific, Inc.; magnification, x200).

Cell lines and cell culture. The two human gastric cancer cell lines HGC-27 (adherent cells) and MKN45 (semi-suspension cells) were used in the present study and purchased from The Cell Bank of Type Culture Collection of The Chinese Academy of Sciences). Cells were cultured in RPMI-1640 complete medium (Corning) supplemented with 10\% FBS (Biological Industries), $100 \mathrm{U} / \mathrm{ml}$ penicillin and $100 \mu \mathrm{g} / \mathrm{ml}$ streptomycin and placed at $37^{\circ} \mathrm{C}$ in a humidified incubator containing $5 \% \mathrm{CO}_{2}$ (Thermo Direct Heat $\mathrm{CO}_{2}$; Thermo Fisher Scientific, Inc.).

Construction and transfection of short hairpin RNA (shRNA) and overexpression vector using a lentivirus. For knockdown and overexpression of FHOD1, lentiviral human FHOD1 shRNA and overexpression vectors were constructed, respectively. The synthesized FHOD1 targeted knockdown and overexpression sequences were cloned into the lentiviral vectors pGL3-U6-enhanced green fluorescence protein (EGFP; TsingKe Biological Technology) and pGV208-EGFP (Shanghai GeneChem Co., Ltd.), respectively. After being incubated at $37^{\circ} \mathrm{C}$ for $2 \mathrm{~h}$, pGL3-U6-shFHOD1 and pGV208-LV-FHOD1 vectors were generated. The empty vector pGV208-EGFP was used as a control plasmid (Shanghai GeneChem Co., Ltd.). The HGC-27 cell line was transfected with pGL3-U6-shFHOD1 and pGV208-LV-FHOD1. The MKN45 cell line was transfected with pGV208-EGFP and pGV208-LV-FHOD1. All transfections were carried out with 1:50 diluted HitransG P (Shanghai GeneChem Co., Ltd.) as transfection reagent. To establish a stable cell line, $72 \mathrm{~h}$ after the lentiviral vectors were transfected, $2 \mu \mathrm{g} / \mathrm{ml}$ puromycin was added to the medium and cells were cultured for 1 week. Knockdown and overexpression efficiency were verified via reverse transcription-quantitative (RT-q)PCR and western blotting.

$R T-q P C R$. TRIzol $^{\circledR}$ reagent (Invitrogen; Thermo Fisher Scientific, Inc.) was used to extract total RNA from HGC-27 and MKN45 cells according to manufacturer's instructions. Total RNA was diluted to $1 \mu \mathrm{g} / 16 \mu \mathrm{l}$ with $4 \mu \mathrm{l} 4 \mathrm{X}$ gDNA wiper mix (Vazyme Biotech Co., Ltd.) and RNase-free $\mathrm{H}_{2} \mathrm{O}$ (Vazyme Biotech Co., Ltd.) at $4^{\circ} \mathrm{C}$ for 2 min to remove genomic DNA. 
Then, $4 \mu 1$ 5X HiScript III qRT SuperMix (Vazyme Biotech Co., Ltd.) was added for RT. The RT conditions were as follows: $\mathrm{RT}$ at $37^{\circ} \mathrm{C}$ for $15 \mathrm{~min}$ and termination at $85^{\circ} \mathrm{C}$ for $5 \mathrm{sec}$. The sequences of the primers designed from NCBI Primer Blast (https://www.ncbi.nlm.nih.gov/tools/primer-blast/index. cgi?LINK_LOC=BlastHome) and were as follows: FHOD1 forward, 5'-CCTCAGCTGACACCTCCAG-3' and reverse, 5'-CAGCGCAACCTGCTTCTC-3'; GAPDH forward, 5'-AGA TCATCAGCAATGCCTCCT-3' and reverse, 5'-TGGTCATGA GTCCTTCCACG-3'; COL1A1 forward, 5'-GATTCCCTG GACCTAAAGGTGC-3' and reverse, 5'-AGCCTCTCCATC TTTGCCAGCA-3'; and COL18A1 forward, 5'-AAGGACGAG CTGCTGTTTCC-3' and reverse, 5'-TTGCCGTCAAAGGAG AAGATG-3'. FHOD1, COL1A1 and COL18A1 expression levels were normalized to those of GAPDH. The SYBR Green PCR MasterMix (Vazyme Biotech Co., Ltd.) and Lightcycle96 system (Roche Applied Science) were used for RT-qPCR. The thermocycling conditions were as follows: Initial denaturation at $95^{\circ} \mathrm{C}$ for $30 \mathrm{sec}$, followed by 40 cycles of PCR amplification at $95^{\circ} \mathrm{C}$ for $10 \mathrm{sec}$ and annealing/elongation at $60^{\circ} \mathrm{C}$ for $30 \mathrm{sec}$. The melting curve was generated at $95^{\circ} \mathrm{C}$ for $15 \mathrm{sec}, 60^{\circ} \mathrm{C}$ for $60 \mathrm{sec}$ and $95^{\circ} \mathrm{C}$ for $15 \mathrm{sec}$ after the thermocycling. The $\mathrm{Cq}$ value calculation and the transcriptional level analysis of the samples were performed using the $2^{-\Delta \Delta \mathrm{Cq}}$ method (19).

Western blotting. HGC-27 and MKN45 cells were lysed using RIPA buffer (Beyotime Institute of Biotechnology) supplemented with a protease and phosphatase inhibitor cocktail (No. 4693116001 Roche Diagnostics GmbH) and phenylmethylsulphonyl fluoride (Biosharp Life Sciences). The cells were lysed at $4^{\circ} \mathrm{C}$ for $30 \mathrm{~min}$ and centrifugated at $14,000 \mathrm{x} \mathrm{g}$ for $30 \mathrm{~min}$ at $4^{\circ} \mathrm{C}$. The supernatant containing proteins was collected. Protein concentration was determined using the BCA method with a KGPBCA kit (cat. no. E162-01; Nanjing KeyGen Biotech Co., Ltd.). Proteins ( $20 \mu \mathrm{g} /$ lane) were separated by 7.5\% PAGE Gel Fast Preparation Kit (EpiZyme) with corresponding protein size standards and transferred onto PVDF membranes. Membranes were blocked with 5\% skimmed milk at room temperature for $2 \mathrm{~h}$. Membranes were incubated with anti-FHOD1 polyclonal mouse antibody (cat. no. ab73443; polyclonal; 1:1,000; anti-mouse; Abcam) at $4^{\circ} \mathrm{C}$ overnight, and then with an HRP-labeled horse anti-mouse antibody (cat. no. 7076; 1:3,000; anti-mouse IgG, HRP-linked Antibody; Cell Signaling Technology, Inc.) at room temperature for $2 \mathrm{~h}$. Enhanced chemiluminescence reagent (Nanjing KeyGen Biotech Co., Ltd.) was used to detect the signal on the membrane.

Cell colony assay. HGC-27 cells transfected with shFHOD1 or LV-FHOD1 were seeded in 6-well plates at the density of 1,000 cells/well $24 \mathrm{~h}$ after transfection, and the medium was replaced every 3 days. Two weeks later, cells were fixed with $100 \%$ methanol for $15 \mathrm{~min}$ and stained with $0.1 \%$ crystal violet for $15 \mathrm{~min}$ (both at room temperature). Each clone (containing $\geq 50$ cells) was manually counted under a x40 magnifying glass. Each experiment was repeated three times.

Cell counting kit (CCK)-8 cell proliferation assay. A CCK-8 (Dojindo Molecular Technologies, Inc.) assay was used to determine GC cell viability. Briefly, cells were seeded into 96-well plates at the density of $3 \times 10^{3}$ cells/well and cultured for $24 \mathrm{~h}$. Then, $10 \mu \mathrm{l} \mathrm{CCK}-8$ reagent was added and cells were incubated at $37^{\circ} \mathrm{C}$ and $5 \% \mathrm{CO}_{2}$ for $1 \mathrm{~h}$. The absorbance was determined at $450 \mathrm{~nm}$ using a plate reader. A growth curve was drawn with time as the horizontal axis and absorbance as the vertical axis.

Cell cycle analysis. $\mathrm{GC}$ cells were trypsinized at $37^{\circ} \mathrm{C}$ for 5 min and washed twice with PBS (cat. no. BL302A; Biosharp Life Sciences). The cell suspension was diluted to the density of $1 \times 10^{6}$ cells $/ \mathrm{ml}$. Subsequently, $1 \mathrm{ml}$ cell suspension was centrifuged at $300 \mathrm{xg}$ for $5 \mathrm{~min}$, the supernatant was discarded and the pellet was mixed with $500 \mu \mathrm{l}$ of $70 \%$ ethanol at $4^{\circ} \mathrm{C}$ overnight. After immobilization, the cells were incubated with $500 \mu \mathrm{l}$ propidium iodide (PI)/RNase A working solution (cat. no. KGA512; Nanjing KeyGen Biotech Co., Ltd.). The DNA content stained with PI was detected by BD FACSCanto II flow cytometer (BD Biosciences) at the peak of $536 \mathrm{~nm}$. Furthermore, the cell cycle distribution was analyzed via flow cytometry and BD FACSDiva software (v9.0; BD Biosciences) was used to analyze the percentage of each cellular population.

Transwell invasion and migration assay. Transwell invasion and migration detections were performed using a Transwell polycarbonate membrane (Corning), which was previously either coated with Matrigel (Corning) or untreated. The bottom chamber was filled with $500 \mu 1$ RPMI-1640 supplemented with $20 \%$ FBS. Then, the transfected HGC-27 cells $\left(1.0 \times 10^{5}\right.$ cells $\left./ \mathrm{ml}\right)$ were seeded into the upper chamber and cultured with FBS-free RPMI-1640 at $37^{\circ} \mathrm{C}$ in a humidified incubator containing $5 \% \mathrm{CO}_{2}$. After $24 \mathrm{~h}$ culture, the remaining cells on the upper surface were mechanically removed. Then, the membranes were cleaned, immobilized in methanol at room temperature for $15 \mathrm{~min}$ and stained with methyl violet at room temperature for $15 \mathrm{~min}$ (Tianjin Institute of Chemical Reagents). Finally, the number of cells that have invaded the lower chamber was determined using a fluorescence inversion microscope (IX51; Olympus Corporation; magnification, $\mathrm{x} 100$ ) in five bright fields to count the number of cells that have migrated through the membrane (ImageJ software; v1.52a; National Institutes of Health).

Cell wound scratch assay. The cell migratory ability was detected using a cell wound scratch assay. Stably transfected GC cells were cultured in 6 -well plates $\left(5 \times 10^{5}\right.$ cells/well). The following day, when $\sim 100 \%$ of the surface was covered with cells, a straight scratch was made to the monolayer with a sterile pipette tip. After washing with PBS twice, cells were cultured for $24 \mathrm{~h}$ in RPMI-1640 medium containing 1\% FBS. The wounds were observed under a $\times 40$ magnification of a fluorescence inversion microscope at 0 and $24 \mathrm{~h}$. The wound width was measured as follows: Migration distance $=$ scratch distance at $0 \mathrm{~h}$-scratch distance at $24 \mathrm{~h}$ (ImageJ software; v1.52a; National Institutes of Health).

Soft agar assay. Agar (Biofroxx; neoFROXX GmbH) was prepared as a $1.2 \%$ solution in normal saline. The $0.6 \%$ agar/medium base layer was added to a 6 -well cell culture plate (Corning) to prevent cell attachment to the plastic base and formation of a monolayer. Cells in the logarithmic phase 
A

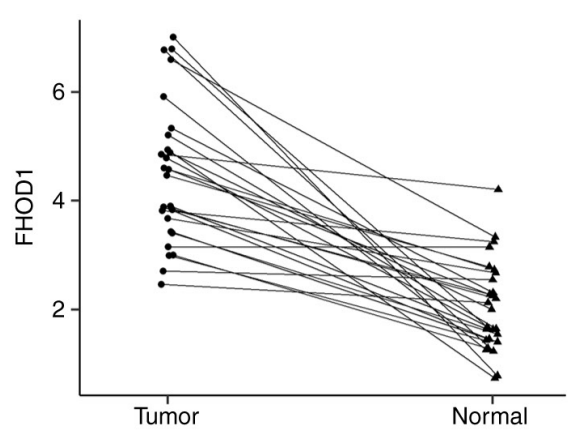

B

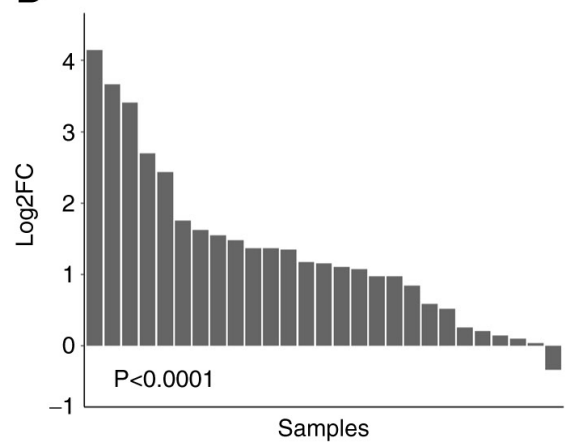

C
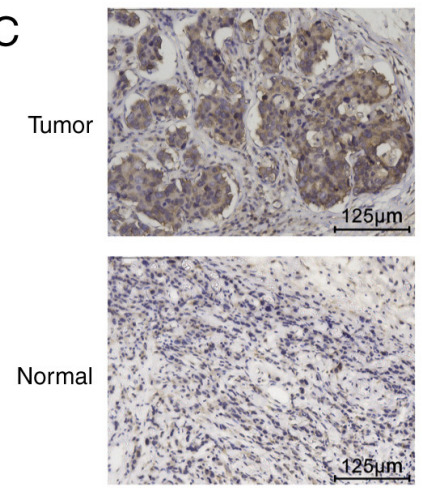

Figure 1. FHOD1 is upregulated in tumor tissues compared with non-tumor tissues in GC. (A) Expression level of FHOD1 in GC tissues compared with adjacent non-tumor tissues in The Cancer Genome Atlas dataset. (B) Log2FC of the expression level of FHOD1 in GC tissues compared with adjacent non-tumor tissues. (C) Expression level of FHOD1 in GC tissues compared with adjacent non-tumor tissues based on immunohistochemistry (magnification, x200). GC, gastric cancer; FHOD1, formin homology 2 domain containing 1 .

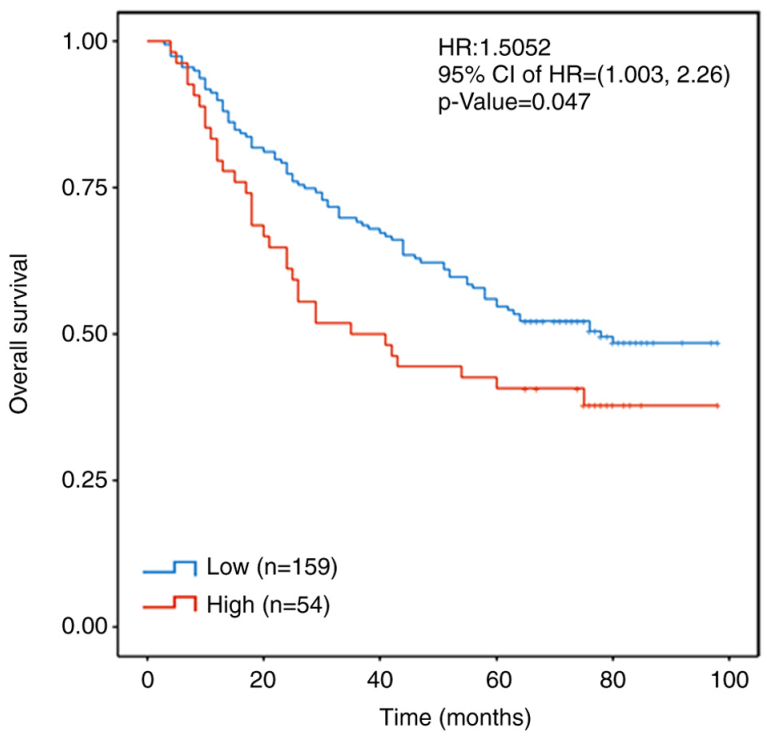

Figure 2. Prognostic value of FHOD1 expression for patients with GC was assessed using Kaplan-Meier analysis. Original expression level data were from our previous panomics-based experiments (18). GC, gastric cancer; FHOD1, formin homology 2 domain containing 1; HR, hazard ratio.

were detached and a $1 \times 10^{4}$ cells $/ \mathrm{ml}$ cell suspension was prepared. The $0.3 \%$ agar upper layer was prepared by mixing $0.6 \%$ low-melting-point agarose with 2-fold cell medium at 1:1 (volume). Subsequently, $1 \mathrm{ml}$ upper agarose mixed with $100 \mu 1$ single-cell suspension $(\sim 1,000$ cells) were added to each well and solidified at room temperature. Cells were cultured at $37^{\circ} \mathrm{C}$ in a humidified incubator containing $5 \% \mathrm{CO}_{2}$ for $1-2$ weeks. Cells were visualized under an inverted microscope for colony counting (only colonies with $>50$ cells each were counted; magnification, $\mathrm{x} 100)$.

Co-expression analysis of FHOD1. Co-expression analysis was performed in TCGA stomach adenocarcinoma dataset using cBioPortal (http://www.cbioportal.org/, TCGA-STAD). WebGestalt (a web-based Gene Set Analysis Toolkit; http://www.webgestalt.org/option.php) was used to assess the enriched Gene Ontology (GO) terms within the gene list of FHOD1 co-expressed genes (FDR<0.05).
3D spheroid culture. HGC-27 and MKN45 cells were cultured in DMEM supplemented with 10\% FBS. To form spheroids, cells were trypsinized at $37^{\circ} \mathrm{C}$ for $5 \mathrm{~min}$ and diluted at 10 cells $/ \mathrm{ml}$, and the cell suspension was added into Corning ${ }^{\circledR}$ Spheroid Microplates (96-well plate; Corning; $100 \mu \mathrm{l} /$ well). Each sample was evaluated in octuplicate. After incubation in a humidified incubator containing $5 \% \mathrm{CO}_{2}$ at $37^{\circ} \mathrm{C}$ for 15 days, total RNA was extracted from spheroids and RT-qPCR was conducted to detect the levels of ECM-related genes (COL1A1 and COL18A1). The RNA extraction and RT-qPCR methods used to detect the ECM-related genes were the same as aforementioned.

Statistical analysis. SPSS software (version 19.0; IBM Corp.) was used for data analysis. Data were presented as the means \pm standard error of the mean of three independent experiments. Unpaired two-tailed Student's t-test or one-way ANOVA followed by Bonferroni's post hoc test were used to analyze differences among the variables. One-way ANOVA followed by Dunnett's post hoc test was employed to analyze differences between multiple sets of data. $\mathrm{P}<0.05$ was considered to indicate a statistically significant difference. The prognostic significance of FHOD1 and overall survival of patients with GC was assessed using the Kaplan-Meier analysis. Patients were stratified based on the FHOD1 expression Z-Score. The cutoff point of Z-Score was-0.267 (optimal cutoff by the maximally selected log-rank statistics).

\section{Results}

FHOD1 expression is elevated in patients with GC. According to TCGA database, the mRNA expression profiles for a total of 27 pairs of GC and adjacent non-tumor tissues were evaluated. A paired samples line graph (Fig. 1A) and bar graph (Fig. 1B) were used to analyze the differences in the expression level of FHOD1 between GC and adjacent non-tumor tissues. The results demonstrated that FHOD1 expression was significantly upregulated in GC tissues $(\mathrm{P}<0.0001)$ compared with adjacent non-tumor tissues. Furthermore, IHC was performed in 30 pairs of human GC samples to further confirm this result. The results demonstrated that FHOD1 expression level was higher in GC tissues compared with adjacent non-tumor 
A

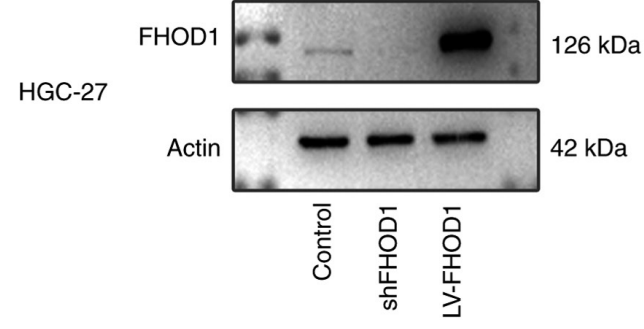

B

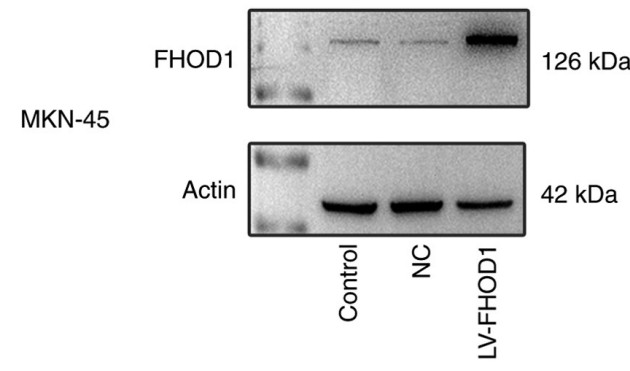

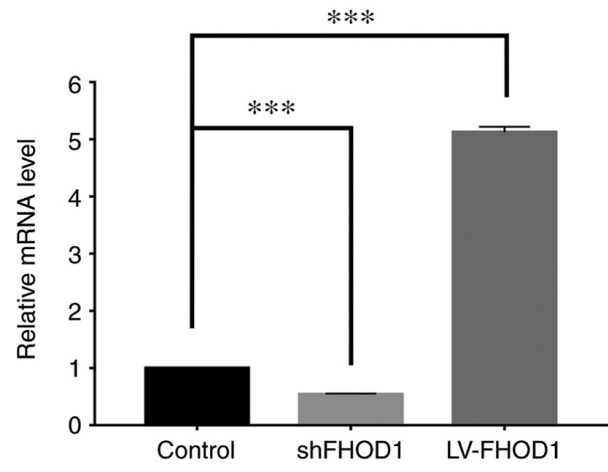

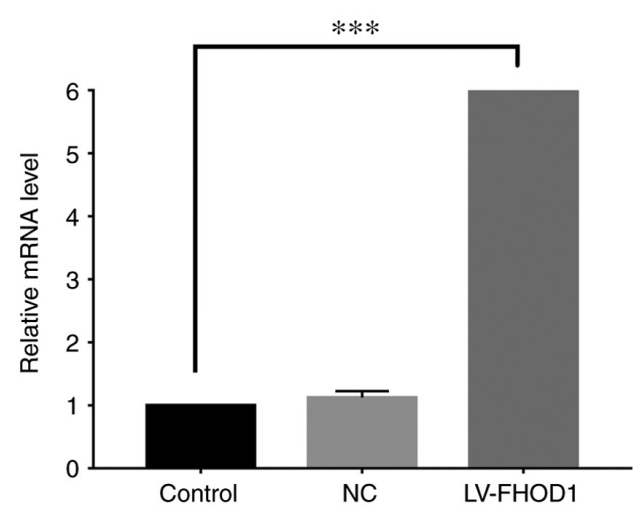

Figure 3. FHOD1 expression levels in HGC27 cells after shFHOD1 or LV-FHOD1 virus transfection, and in MKN45 cells after LV-FHOD1 virus transfection. (A) FHOD1 mRNA and protein expression levels in HGC27 cells were detected via RT-qPCR (left) and western blotting (right). (B) FHOD1 mRNA and protein expression levels in MKN45 cells were detected via RT-qPCR (left) and western blotting (right). ${ }^{* * *} \mathrm{P}<0.001$. FHOD1, formin homology 2 domain containing 1; RT-qPCR, reverse transcription-quantitative PCR; sh, short hairpin RNA. Control, untransfected cells; NC, negative control; LV, lentivirus.

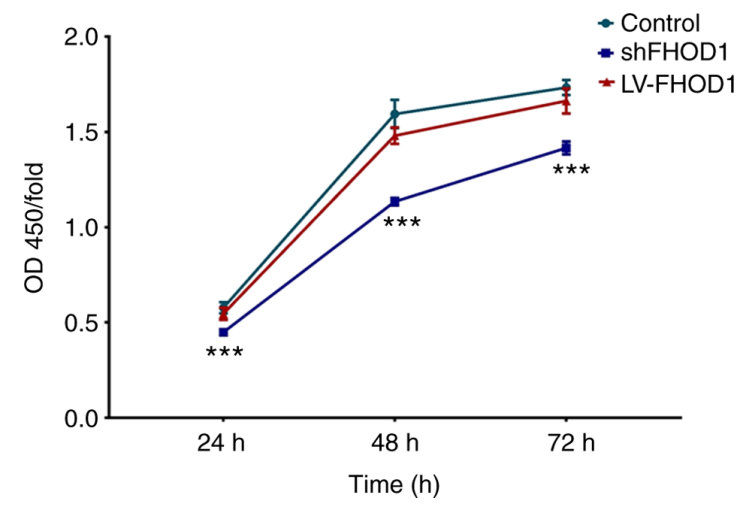

Figure 4. Proliferation of gastric cancer cells after knockdown and overexpression of FHOD1. Cell Counting Kit- 8 cell proliferation assay demonstrated that the proliferative rate was significantly decreased at each time point after FHOD1 knockdown in HGC-27 cells. ${ }^{* * *} \mathrm{P}<0.001$. FHOD1, formin homology 2 domain containing 1 ; sh, short hairpin; OD, optical density; LV, lentivirus.

gastric tissues from patients enrolled at Nanjing Drum Tower Hospital (Fig. 1C). Taken together, these findings suggest that FHOD1 was upregulated in GC.

FHOD1 expression is inversely associated with prognosis in patients with $G C$. In order to further evaluate the significance of FHOD1 in the development of GC, Kaplan-Meier analysis was performed on the transcriptome data of 203 patients with GC, which were obtained from our previous study using the Panomics technique designed to evaluate the prognostic value of a multigene panel for patients with GC in a hospital cohort (18).
Patients were stratified according to the Z-scores of FHOD1 expression. If the Z-Score of FHOD1 expression was >-0.267 (the normal distribution limit of the right tail was 0.95), this was defined as a high FHOD1 expression. The results demonstrated that a high expression level of FHOD1 was associated with a shortened overall survival in patients with GC (Fig. 2).

Efficiency of FHOD1 knockdown and overexpression using lentivirus-mediated vectors in HGC27 and MKN45 cells. RT-qPCR was performed to detect FHOD1 expression and the transfection efficiency in human GC HGC-27 and MKN45 cells. In both cell lines, FHOD1 was moderately expressed, indicating that these two cell lines were suitable for FHOD1 knockdown and overexpression. Lentivirus-mediated shFHOD1 and LV-FHOD1 were designed and used to infect HGC-27 and MKN45 cells. The results demonstrated that FHOD1 mRNA expression in the shFHOD1 group in HGC-27 cells was 50\% lower compared with that in the untransfected group, and the FHOD1 mRNA expression in the LV-FHOD1 group was 5-fold higher compared with that in the untransfected group (Fig. 3A). In MKN45 cells, FHOD1 expression in the negative control group (transfected with control vector) was similar to the untransfected group, while FHOD1 expression in the LV-FHOD1 group was 5.5-fold higher compared with that in the untransfected cells (Fig. 3B). Furthermore, data from western blotting supported the findings from RT-qPCR (Fig. 3A and B).

Effect of FHOD1 knockdown or overexpression on the proliferation of GC cells. In order to examine the possible change in cell proliferation according to FHOD1 expression, 

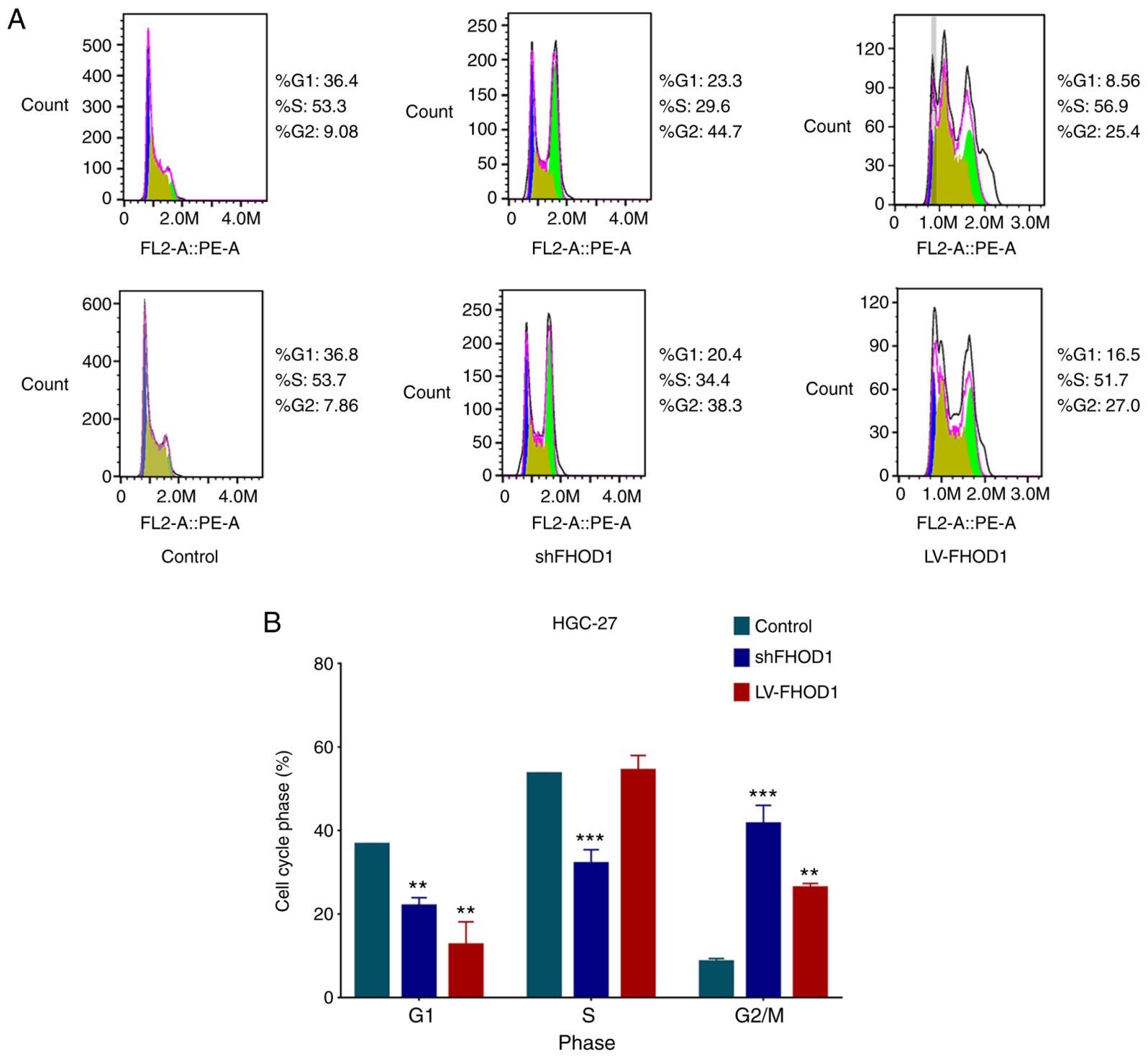

Figure 5. Cell cycle distribution measured via flow cytometry in GC cells after FHOD1 knockdown and overexpression. (A) Representative plots for the Cell Cycle Phase detection for each of the 3 groups. (B) Quantification of GC cell cycle distribution measured via flow cytometry in GC cells after knockdown and overexpression of FHOD1. Proportion of cells in S phase after FHOD1 knockdown was significantly decreased and that of cells in G1 phase after FHOD1 overexpression was markedly decreased. ${ }^{* *} \mathrm{P}<0.01$ and ${ }^{* * * *} \mathrm{P}<0.001$. FHOD1, formin homology 2 domain containing 1 ; sh, short hairpin; GC, gastric cancer; LV, lentivirus.

certain assays were performed to evaluate the cell proliferation, cell cycle and colony formation.

In the CCK-8 cell proliferation assay, knockdown of FHOD1 induced a significant inhibitory effect on GC cell proliferation at 24, 48 and $72 \mathrm{~h}(\mathrm{P}<0.001$; Fig. 4).

Cell cycle was analyzed via flow cytometry in transfected and control GC cells. As presented in Fig. 5, the $\mathrm{G}_{1}-\mathrm{S}$ phase transition in the cell cycle was inhibited, the $\mathrm{S}$ ratio was significantly decreased, and the $\mathrm{G}_{2} / \mathrm{M}$ ratio was significantly increased in HGC-27 cells following FHOD1 knockdown compared with control cells. Furthermore, the $\mathrm{G}_{1}$ ratio was significantly reduced, and the $\mathrm{G}_{2} / \mathrm{M}$ ratio was significantly increased in HGC-27 cells overexpressing FHOD1. These results indicated that the knockdown and overexpression of FHOD1 could affect the cell cycle of HGC-27 cells, primarily by affecting the $\mathrm{G}_{1}-S$ phase transition and causing $\mathrm{G}_{2} / \mathrm{M}$ arrest. It is known that $\mathrm{G}_{1}-\mathrm{S}$ transition and $\mathrm{G}_{2} / \mathrm{M}$ arrest are associated with the oncogenic process (20).
Subsequently, a colony formation experiment was performed (Fig. 6) and the results demonstrated that FHOD1 knockdown could significantly inhibit the proliferation of HGC-27 compared with LV-FHOD1-transfected group and the control cells. This finding suggested that FHOD1 knockdown may significantly inhibit the proliferative ability of HGC-27 cells $(\mathrm{P}<0.01)$.

Effect of FHODI knockdown or overexpression on the migratory ability of GC cells. The effects of FHOD1 knockdown and overexpression on the migratory ability of GC cells were examined using a cell wound scratch assay. As presented in Fig. 7A and B, the wound healing of FHOD1-knockdown HGC-27 cells was significantly delayed compared with FHOD1-overexpressed cells and non-transfected cells (Fig. 7A). Conversely, the wound healing rate was significantly increased in FHOD1-overexpressing cells (Fig. 7A). 

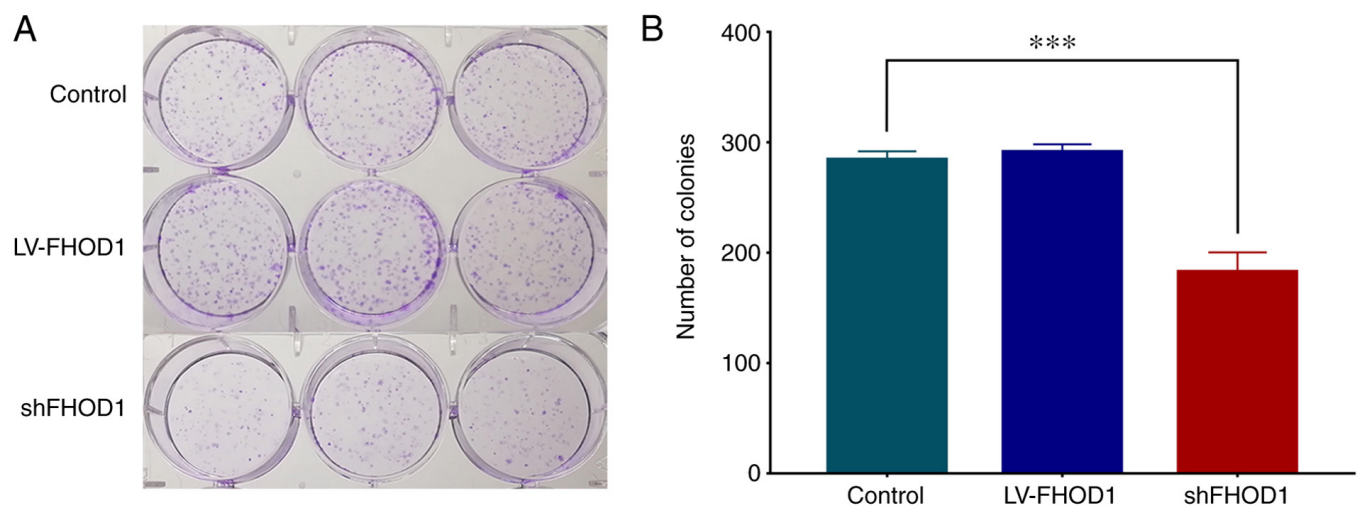

Figure 6. Efficiency of HGC-27 colony formation following shFHOD1 or LV-FHOD1 transfection for 2 weeks. (A) Cell colony growth of HGC-27 cells in petri dishes in the control, shFHOD1 and LV-FHOD1 groups. (B) Quantification of cell colony numbers of HGC-27 cells in the control, shFHOD1 and LV-FHOD1 groups. ${ }^{* * *} \mathrm{P}<0.001$. sh, short hairpin RNA; FHOD1, formin homology 2 domain containing 1; sh, short hairpin; LV, lentivirus.
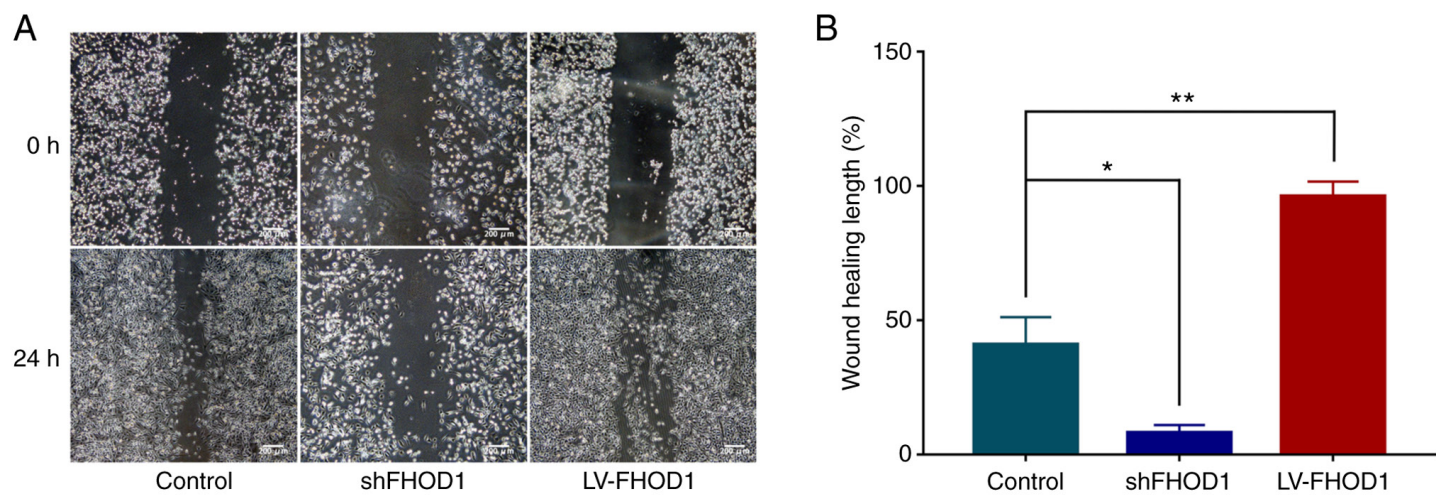

Figure 7. Migratory ability of HGC-27 cells after FHOD1 knockdown and overexpression. (A) Migratory potential of stably transfected HGC27 cells was examined using a cell wound scratch assay. Images were captured at time intervals of 0 and $24 \mathrm{~h}$ (magnification, x40). (B) Percentage of migration was statistically analyzed. ${ }^{*} \mathrm{P}<0.05$ and ${ }^{* *} \mathrm{P}<0.01$. FHOD1, formin homology 2 domain containing 1 ; sh, short hairpin; LV, lentivirus.

A

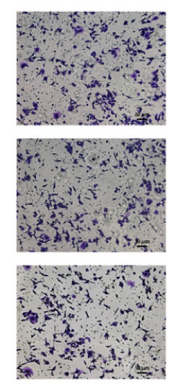

Control

B

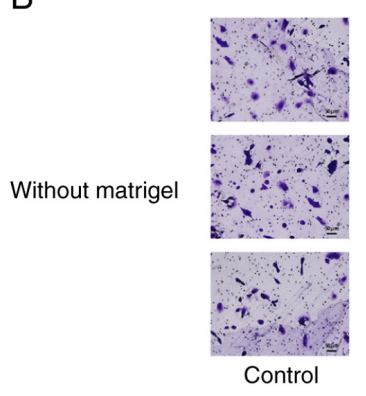

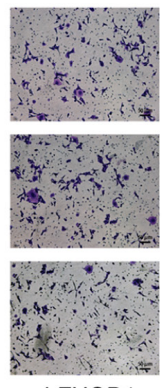

shFHOD1

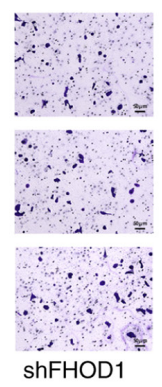

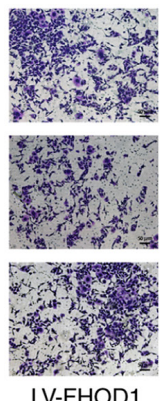

LV-FHOD1

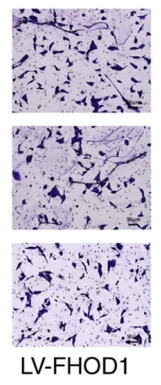

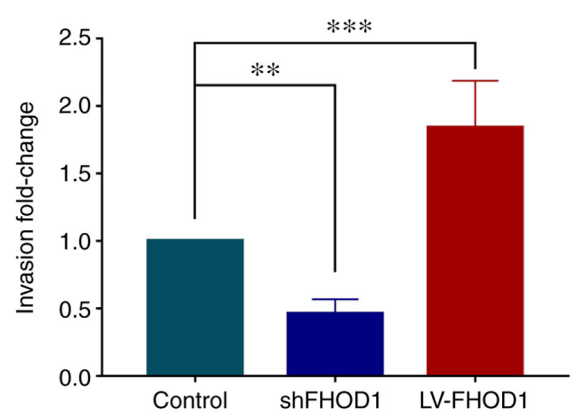

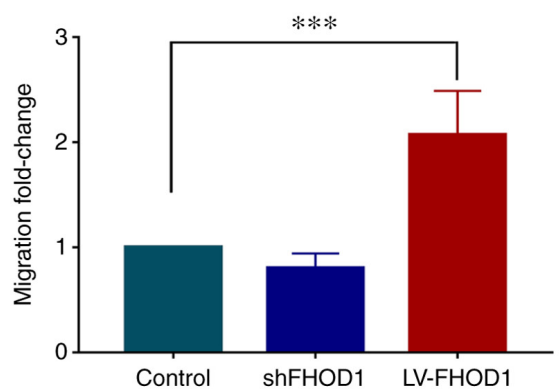

Figure 8. Invasive ability of GC cells after knockdown and overexpression of FHOD1 with or without Matrigel. (A) Left: The number of HGC-27 cells crossing the basement membrane with Matrigel (magnification, x100). Right: Quantification of GC cells crossing the basement membrane after knockdown and overexpression of FHOD1. (B) Left: The number of HGC-27 cells crossing the basement membrane without Matrigel (magnification, x100). Right: Quantification of GC cells crossing the basement membrane after knockdown and overexpression of FHOD1. ${ }^{* *} \mathrm{P}<0.01$ and ${ }^{* * * *} \mathrm{P}<0.001$. FHOD1, formin homology 2 domain containing 1; GC, gastric cancer; sh, short hairpin; LV, lentivirus. 

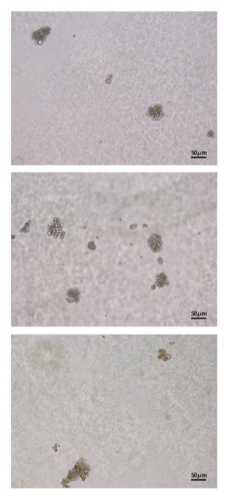

Control
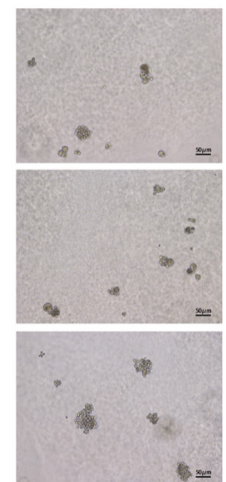

NC
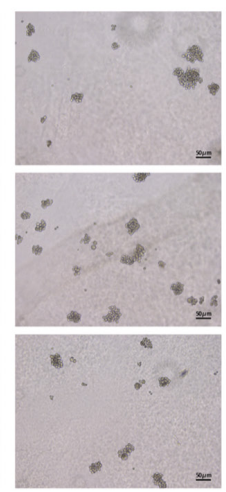

LV-FHOD1

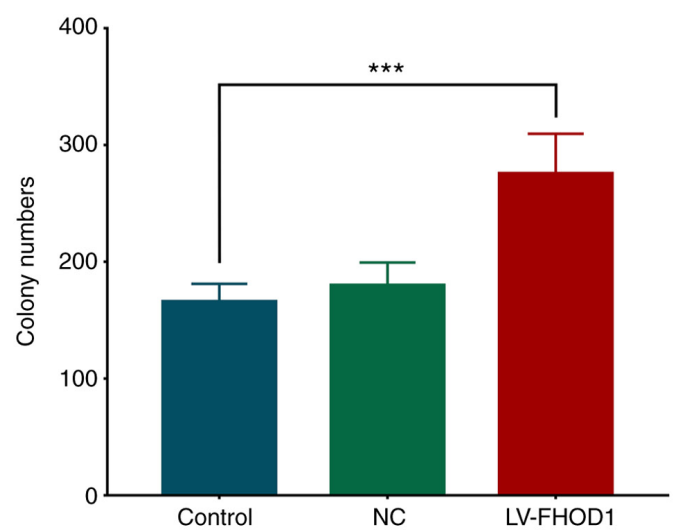

Figure 9. Viability of MKN45 cells after FHOD1 overexpression was analyzed using a soft agar colony formation assay (magnification, $\mathrm{x} 100)$. ${ }^{* * *} \mathrm{P}<0.001$ FHOD1, formin homology 2 domain containing 1; NC, negative control; LV, lentivirus.

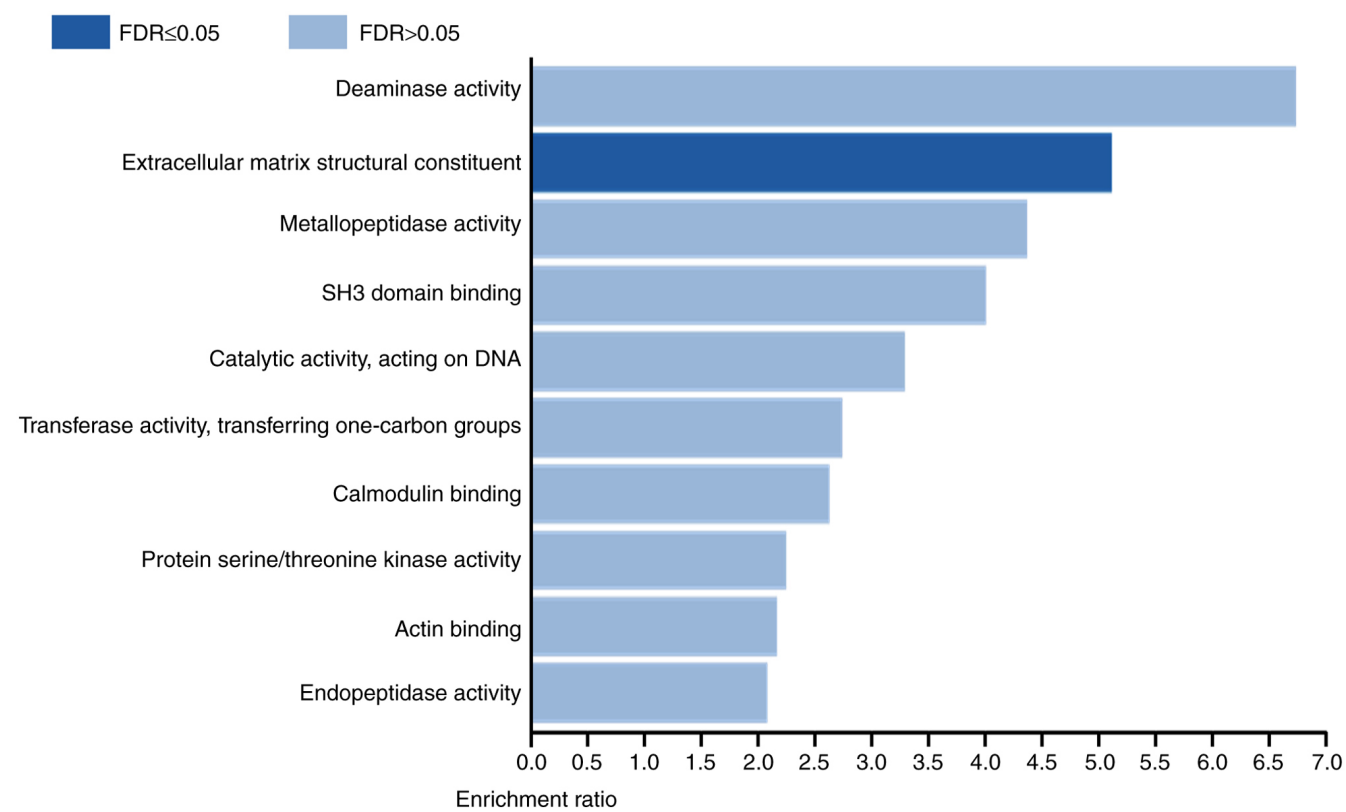

Figure 10. Functional enrichment analysis of genes positively co-expressed with FHOD1 using WebGestalt (Web-based Gene Set analysis Toolkit). FHOD1, formin homology 2 domain containing 1 .

A

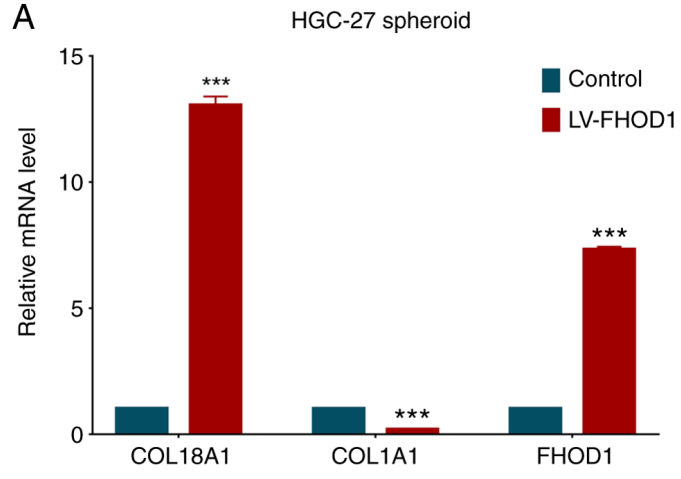

B

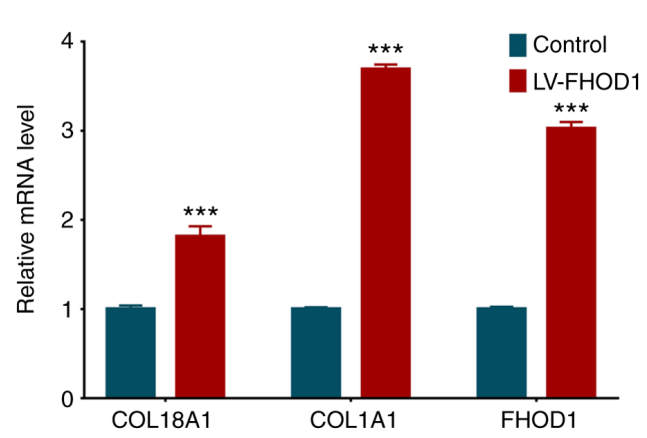

Figure 11. Expression levels of ECM-related genes and FHOD1 in HGC-27 and MKN-45 spheroids. (A) COL1A1 and COL18A1 expression in HGC-27 spheroids after FHOD1 overexpression. (B) COL1A1 and COL18A1 expression in MKN-45 spheroids after FHOD1 overexpression. ${ }^{* * *} \mathrm{P}<0.001$. FHOD1, formin homology 2 domain containing 1; COL1A1, collagen type I alpha 1 chain; COL18A1, collagen type XVIII alpha 1 chain.

In vitro migratory and invasive abilities. The results demonstrated that the invasive ability of HGC-27 cells following
FHOD1 knockdown was significantly decreased compared with control group; however, it was significantly increased 
after FHOD1 overexpression (Fig. 8A). In addition, knockdown of FHOD1 notably inhibited the migratory ability of HGC-27 cells compared with the control cells; however, it was significantly increased after FHOD1 overexpression (Fig. 8B).

FHOD1 promotes the proliferation of MKN45 cells. The results from soft agar colony formation assay results demonstrated that the number of colonies 14 days after FHOD1 overexpression was significantly higher compared with that of the empty vector and untransfected HGC-27 cells (Fig. 9).

Genes positively co-expressed with FHODI are enriched in the molecular function of extracellular matrix (ECM) structural constituent. To understand the role of FHOD1, co-expression analysis was performed in TCGA stomach adenocarcinoma data using cBioPortal. It was demonstrated that the expression levels of 159 genes were positively correlated, while the expression levels of 75 genes were negatively correlated with the expression level of FHOD1 $\left(|\mathrm{r}| \geq 0.25\right.$; adjusted $\mathrm{P}<1.00 \times 10^{-4}$; Table SI). Subsequently, functional enrichment analysis of the genes co-expressed with FHOD1 was conducted using WebGestalt. The results demonstrated that genes which expression levels were positively correlated with FHOD1 were enriched in the Gene Ontology term 'Extracellular matrix structural constituent', which contributes to the structural integrity of the ECM (Fig. 10). No functional enrichment was identified for genes negatively correlated with FHOD1. These results suggested that FHOD1 may contribute to GC development and progression by regulating the structural integrity of the ECM.

ECM-related gene expression is regulated by FHODI in a $3 D$ spheroid culture. To determine whether FHOD1 regulates the expression of ECM-related genes, such as COL1A1 and COL18A1, which were found to be positively correlated with FHOD1 expression in GC following bioinformatics analysis, HGC-27 and MKN45 cells overexpressing FHOD1 were cultured in vitro to form spheroids and total RNA was extracted. The expression levels of COL1A1 and COL18A1, along with FHOD1, were measured by RT-qPCR. As presented in Fig. 11, FHOD1 overexpression significantly increased the expression of COL18A1 in both cell lines, while FHOD1 overexpression only significantly upregulated COL1A1 in MKN45 cells. These results suggested that FHOD1 may regulate the expression of ECM-related genes to ensure the structural integrity of the ECM.

\section{Discussion}

The development of GC is considered as a multifactorial process involving cell proliferation, tumor growth, ECM degradation, cell adhesion and motility, and angiogenesis (21-25). The regulation of these cellular processes involves oncogenes and tumor suppressor genes, and their corresponding encoded products and pathways. The present study aimed, therefore, to investigate the biological role of FHOD1 in human GC cells.

FHOD1 is a member of the formin family. Formins are highly conserved actin nucleating proteins found in all eukaryotic cells. The activity of formins is regulated by Rho GTPases, which are molecular switched that reshape the cytoskeleton in different intercellular spaces $(13,14)$. Rho GTPases serve a unique role in controlling the assembly of stress fibers, the formation of adhesion foci and the movement pattern of cancer cells (26,27). Gardberg et al (28) reported that FHOD1 is upregulated in the oral squamous cell carcinoma, which affects the morphological and functional characteristics of EMT, including actin tissue remodeling, cell migration and the capability to degrade ECM. It has been demonstrated that FHOD1 may mediate the changes and migration characteristics of the cytoskeleton during cancer-related EMT in triple-negative breast cancer and oral squamous cell carcinoma (28-30). FHOD1 was reported to be involved in early cell migration or invasion and is upregulated in clinical tumor tissues of basal-like breast cancer (25). In addition, FHOD1 is overexpressed in glioblastoma and melanoma tissues, and is also involved in increased migration and invasion of HGC-27 in vitro $(11,31)$. However, to the best of our knowledge, the expression of FHOD1 in human GC tissues is unknown.

In the present study, the analysis of TCGA transcriptome data and results from IHC on GC human samples demonstrated that FHOD1 expression levels were significantly upregulated in GC tissues compared with normal tissues. Furthermore, the high expression level of FHOD1 was significantly associated with the poor prognosis of patients with GC. These findings suggested that FHOD1 may serve an important biological role in the progression of the GC.

Because the role of FHOD1 has not yet been reported in human GC, the present study investigated the effects of FHOD1 knockdown or overexpression on certain important cellular processes in two gastric cancer cell lines. The results indicated that cell proliferation was inhibited following FHOD1 knockdown in HGC-27 cells. Furthermore, after FHOD1 knockdown, the cell cycle was blocked. Conversely, FHOD1 overexpression in HGC-27 cells promoted soft-agar colony formation, migration and invasion, but did not affect proliferation. The same results were verified in MKN45 GC cells overexpressing FHOD1. Previous studies have demonstrated that FHOD1 serves a critical role in the structural and functional changes of microfilaments, which is necessary for the transformation of the cell phenotype from epithelial to mesenchymal (28-30). The findings from the present study were consistent with these previous studies, suggesting that FHOD1 may serve a role in GC progression, although it may not regulate cell proliferation.

It has been reported that serum response element (SRE) can be activated by FHOD1 via its effect on the actin cytoskeleton in melanoma cells (31). Deletion of FHOD1 decreases SRE transcription, resulting in cell cycle arrest and decreased cell proliferation $(8,9)$. In the present study, the role of FHOD1 was also evaluated using migration and invasion assays. The cell migratory and invasive abilities were significantly decreased in HGC-27 cells after FHOD1-shRNA transfection and were significantly higher in the LV-FHOD1 group compared with the control. As MKN45 cells possess the characteristic of semi-suspension in culture, these cells were not suitable for certain assays, such as cell wound scratch, colony formation and Transwell invasion assays. However, since MKN45 cells can float in soft agar, they were used for soft agar-based proliferation experiment. Taken together, the present 
findings suggested that FHOD1 may regulate the proliferation, migration and invasion of GC cells in vitro.

The current study presented certain limitations. All experiments were performed using only two GC cell lines. The findings obtained should therefore be verified using additional GC cell lines and normal gastric cell lines in future studies. Furthermore, the tumorigenic effect and tumor-promoting mechanism of FHOD1 will be further verified using genetic manipulation-based approaches to determine whether collagen genes, such as COL1A1 and COL18A1, serve an important role in FHOD1-related gastric tumorigenesis using appropriate model systems. For example, a further study will investigate whether restoration of COL18A1 expression in FHOD1-knockdown cells could rescue their tumorigenic phenotype, and whether COL18A1 knockdown in FHOD1-overexpressing cells decrease tumorigenicity. Using the correct model systems is therefore crucial to investigate the impact of ECM-related functions on tumorigenesis.

In conclusion, the present study demonstrated that FHOD1 was upregulated in GC tissues compared with adjacent normal tissues, and that FHOD1 expression was associated with a poorer overall survival in patients with GC. In human GC cells, FHOD1 knockdown and overexpression could modulate the cell proliferation, migration and invasion in vitro. The co-expression and functional enrichment analyses revealed that genes which expression levels were correlated with FHOD1 were enriched in the GO term of the 'Extracellular matrix structural constituent', suggesting that FHOD1 may serve a role in the regulation of ECM structural integrity. Although the underlying mechanisms remain unknown, the results from the present study suggested that FHOD1 may serve an important role in the occurrence and development of GC and may therefore be considered as a potential target for the treatment of GC.

\section{Acknowledgements}

Not applicable.

\section{Funding}

The present study was supported by the National Natural Science Foundation of China (grant no. 81802388) and the Natural Science Foundation from the Department of Science and Technology of Jiangsu Province (grant no. BK20180120).

\section{Availability of data and materials}

The datasets used and/or analyzed during the present study are available from the corresponding author on reasonable request.

\section{Authors' contributions}

PW designed the experiments and analyzed data. CJ and BY performed the experiments and wrote the manuscript. $\mathrm{XZ}$ was responsible for data analysis and manuscript revision. JHM and $\mathrm{BH}$ analyzed data and edited the manuscript. XZ and PW confirm the authenticity of all the raw data. All authors read and approved the final manuscript.

\section{Ethics approval and consent to participate}

Not applicable.

\section{Patient consent for publication}

Not applicable.

\section{Competing interests}

The authors declare that they have no competing interests.

\section{References}

1. Bray F, Ferlay J, Soerjomataram I, Siegel RL, Torre LA and Jemal A: Global cancer statistics 2018: GLOBOCAN estimates of incidence and mortality worldwide for 36 cancers in 185 countries. CA Cancer J Clin 68: 394-424, 2018.

2. Global Burden of Disease Cancer Collaboration, Fitzmaurice C, Dicker D, Pain A, Hamavid H, Moradi-Lakeh M, MacIntyre MF, Allen C, Hansen G, Woodbrook R, et al: The global burden of cancer 2013. JAMA Oncol 1: 505-527, 2015.

3. International Agency for Research on Cancer (IARC): Cancer Fact Sheets, Digestive organs. IARC, Lyon, 2020. https://gco. iarc.fr/today/fact-sheets-cancer. Accessed December, 2020.

4. National Cancer Institute (NIH): Cancer Stat Facts: Stomach cancer. NIH, Bethesda, MD, 2021. https://seer.cancer.gov/statfacts $/$ html/stomach.html.

5. Bevan S and Houlston RS: Genetic predisposition to gastric cancer. QJM 92: 5-10, 1999.

6. Ushijima T and Sasako M: Focus on gastric cancer. Cancer Cell 5: 121-125, 2004.

7. Wang P, Wang Y, Hang B, Zou X and Mao JH: A novel gene expression-based prognostic scoring system to predict survival in gastric cancer. Oncotarget 7: 55343-55351, 2016.

8. Cui J, Li F, Wang G, Fang X, Puett JD and Xu Y: Gene-expression signatures can distinguish gastric cancer grades and stages. PLoS One 6: e17819, 2011.

9. Mattioni M, Soddu S, Porrello A, D'Alessandro R, Spila A and Guadagni F: Serum anti-p53 antibodies as a useful marker for prognosis of gastric carcinoma. Int J Biol Markers 22: 302-306, 2007.

10. Takeno A, Takemasa I, Doki Y, Yamasaki M, Miyata H, Takiguchi S, Fujiwara Y, Matsubara K and Monden M: Integrative approach for differentially overexpressed genes in gastric cancer by combining large-scale gene expression profiling and network analysis. Br J Cancer 99: 1307-1315, 2008.

11. Heuser VD, Kiviniemi A, Lehtinen L, Munthe S, Kristensen BW, Posti JP, Sipilä JOT, Vuorinen V, Carpén O and Gardberg M: Multiple formin proteins participate in glioblastoma migration. BMC Cancer 20: 710, 2020.

12. Zhao S, Cai J, Zhang X, Cui J and Jiu Y: Different formins restrict localization of distinct tropomyosins on dorsal stress fibers in osteosarcoma cells. Cytoskeleton (Hoboken) 77: 16-24, 2020.

13. Alvarez DE and Agaisse H: A role for the small GTPase Rac1 in vaccinia actin-based motility. Small GTPases 6: 119-122, 2015.

14. Schulte A, Stolp B, Schönichen A, Pylypenko O, Rak A, Fackler OT and Geyer M: The human formin FHOD1 contains a bipartite structure of $\mathrm{FH} 3$ and GTPase-binding domains required for activation. Structure 16: 1313-1323, 2008.

15. Heuser VD, Mansuri N, Mogg J, Kurki S, Repo H, Kronqvist P, Carpén $\mathrm{O}$ and Gardberg M: Formin proteins FHOD1 and INF2 in triple-negative breast cancer: Association with basal markers and functional activities. Breast Cancer (Auckl) 12: 1178223418792247, 2018.

16. Sun BO, Fang Y, Li Z, Chen Z and Xiang J: Role of cellular cytoskeleton in epithelial-mesenchymal transition process during cancer progression. Biomed Rep 3: 603-610, 2015.

17. Li DJ, Feng ZC, Li XR and Hu G: Involvement of methylation-associated silencing of formin 2 in colorectal carcinogenesis. World J Gastroenterol 24: 5013-5024, 2018.

18. Zhu L, Wang H, Jiang C, Li W, Zhai S, Cai X, Wang X, Liao L, Tao F, Jin D, et al: Clinically applicable $53-G e n e$ prognostic assay predicts chemotherapy benefit in gastric cancer: A multicenter study. EBioMedicine 61: 103023, 2020. 
19. Livak KJ and Schmittgen TD: Analysis of relative gene expression data using real-time quantitative PCR and the 2(-Delta Delta C(T)) method. Methods 25: 402-408, 2001.

20. Bertoli C, Skotheim JM and de Bruin RA: Control of cell cycle transcription during G1 and S phases. Nat Rev Mol Cell Biol 14 518-528, 2013

21. Zhang H, Wang Y, Wang Y, Wu D, Lin E and Xia Q: Intratumoral and intertumoral heterogeneity of HER2 immunohistochemical expression in gastric cancer. Pathol Res Pract 216: 153229, 2020.

22. Dai J, Peng T and Yu X: NK6 homeobox 2 regulated gastrokin-2 suppresses gastric cancer cell proliferation and invasion via Akt signaling pathway. Cell Biochem Biophys 79: 123-131, 2021.

23. Han X, Zhang HB, Li XD and Wang ZA: Long non-coding RNA $\mathrm{X}$-inactive-specific transcript contributes to cisplatin resistance in gastric cancer by sponging miR-let-7b. Anticancer Drugs 31: $1018-1025,2020$

24. Gao N, Yang F, Chen S, Wan H, Zhao X and Dong H: The role of TRPV1 ion channels in the suppression of gastric cancer development. J Exp Clin Cancer Res 39: 206, 2020.

25. Li Z, Gao H, Liu Y, Wu H, Li W, Xing Y, Zhang Z and Zhang X Genetic variants in the regulation region of TLR4 reduce the gastric cancer susceptibility. Gene 767: 145181, 2021.

26. Jurmeister S, Baumann M, Balwierz A, Keklikoglou I, Ward A, Uhlmann S, Zhang JD, Wiemann S and Sahin Ö: MicroRNA-200c represses migration and invasion of breast cancer cells by targeting actin-regulatory proteins FHOD1 and PPM1F. Mol Cell Biol 32: 633-651, 2012.
27. Gasteier JE, Madrid R, Krautkrämer E, Schröder S, Muranyi W, Benichou S and Fackler OT: Activation of the Rac-binding partner FHOD1 induces actin stress fibers via a ROCK-dependent mechanism. J Biol Chem 278: 38902-38912, 2003.

28. Gardberg M, Kaipio K, Lehtinen L, Mikkonen P, Heuser VD, Talvinen K, Iljin K, Kampf C, Uhlen M, Grénman R, et al: FHOD1, a formin upregulated in epithelial-mesenchymal transition, participates in cancer cell migration and invasion. PLoS One 8: e74923, 2013.

29. Haraguchi T, Kondo M, Uchikawa R, Kobayashi K, Hiramatsu H, Kobayashi K, Chit UW, Shimizu T and Iba H: Dynamics and plasticity of the epithelial to mesenchymal transition induced by miR-200 family inhibition. Sci Rep 6: 21117, 2016.

30. Perdigão-Henriques R, Petrocca F, Altschuler G, Thomas MP, Le MT, Tan SM, Hide W and Lieberman J: miR-200 promotes the mesenchymal to epithelial transition by suppressing multiple members of the Zeb2 and Snaill transcriptional repressor complexes. Oncogene 35: 158-172, 2016.

31. Peippo M, Gardberg M, Lamminen T, Kaipio K, Carpén O and Heuser VD: FHOD1 formin is upregulated in melanomas and modifies proliferation and tumor growth. Exp Cell Res 350: 267-278, 2017. 